Ann. Biol. anim. Bioch. Biophys., I972, 12 (I), 9I-Io8.

\title{
SYNTHĖSES PROTÉIQUES IN VIVO DANS DIVERS TISSUS DU RAT EN CROISSANCE SOUMIS A UNE RÉDUCTION DE L'APPORT ÉNERGÉTIQUE DE LA RATION
}

\author{
M. ARNAL, G. FAUCONNEAU et Renée PECH \\ avec la collaboration technique d'Évelyne Aurousseau \\ Station d'Étude des Métabolismes, \\ Centre de Recherches de Clermont-Ferrand, I. N. R. A., \\ 63 - Saint-Genès-Champanelle
}

\section{RÉSUMÉ}

Après injection intrapéritonéale de L-lysine- ${ }^{14} \mathrm{C}(\mathrm{U})$ à des rats témoins nourris ad libitum permettant un gain de $6 \mathrm{~g} / \mathrm{j}$ ou soumis à une réduction de $50 \mathrm{p}$. Ioo de l'apport énergétique de la ration $(2,2 \mathrm{~g} / \mathrm{j}$ de gain de poids), la radioactivité de la $\mathrm{L}$-lysine-14 $\mathrm{C}$ est mesurée à des temps variables de 30 à I $500 \mathrm{mn}$ après l'injection dans les fractions acides aminés libres et protéiques des 6 compartiments suivants : sang, muscles des membres postérieurs, appareil digestif, contenu digestif, peau et carcasse. Les synthèses protéiques ont été appréciées dans le muscle, la peau et l'appareil digestif.

En comparaison des rats témoins de même poids ( $200 \mathrm{~g}$ ), les rats carencés en énergie présentent les modifications suivantes :

- la teneur en lysine libre est augmentée dans chacun des compartiments ;

- l'évolution de la radioactivité spécifique de la lysine-1" $\mathrm{C}$ libre est identique à celle des témoins excepté au temps $24^{\circ} \mathrm{mn}$ où elle est plus élevée ;

- la quantité de radioactivité dégagée sous forme de ${ }^{14} \mathrm{CO}_{2}$ est 3 fois plus élevée au bout de 8 heures, ce qui indique une utilisation des chaînes carbonées de la lysine à des fins énergétiques :

- la vitesse d'incorporation de la L-lysine-14C est diminuée dans le muscle, la peau et le sang;

- dans les muscles, la peau et l'appareil digestif, la valeur des synthèses protéiques représente respectivement 60 p. roo, $50 \mathrm{p}$. I00 et $200 \mathrm{p}$. Ioo de celle des témoins ;

- les radioactivités des fractions acido-soluble et insoluble du contenu digestif sont beaucoup plus importantes.

\section{INTRODUCTION}

La mise au point de Munro (I964) et les travaux de WANNEMACHER (I965) mettent en évidence chez le rat la très bonne corrélation existant entre les pertes de protéines et d'acide ribonucléique (ARN) dans le foie, le muscle et la peau sous l'influence d'un régime protéiprive. 
Dans le cas d'animaux en croissance soumis à une carence azotée qualitative : déficit en certains acides aminés indispensables (lysine, thréonine et tryptophane) (Durand et FaUconNeaU, I966; VANDERMEERs-Pire'T et Christophe, I964), une diminution des teneurs en acides désoxyribonucléiques (ADN), ARN et protéines est observée dans la carcasse, le foie et l'intestin grêle. Le rapport $\frac{A R N}{A D N}$, un des critères de l'activité cellulaire, est toujours diminué. A poids égal des animaux, les cellules de ces organes sont moins nombreuses, plus grandes et généralement plus riches en protéines chez les animaux carencés que chez les témoins. Le rôle de l'ARN dans le mécanisme de la synthèse protéique est bien connu et l'on pourrait conclure qu'une diminution de la teneur en ARN entraînerait un ralentissement des synthèses protéiques. Or, l'emploi d'acide aminé radioactif comme précurseur dans le cas de régime protéiprive (GAETANI et al., I963) à bas niveau protéique (WATERLOW et STEPHEN, I968), carencé en un acide aminé indispensable : thréonine (SIDRANSKY et WAGLE, I969; SIDRANSKY et VERNEX, I970) ou en plusieurs acides aminés : lysine, thréonine et tryptophane (VANDERMEERS et VANDERMEERS-PIRET, I967), a permis de montrer que, au contraire, les synthèses protéiques appréciées par incorporation de leucine${ }^{14} \mathrm{C}$ étaient augmentées dans le foie ainsi que dans le pancréas (VANDERMEERS et VANDERMEERS-PIRET, I967). La plupart de ces auteurs démontrent par la même méthode, qu'au niveau du muscle il y a bien un ralentissement de l'incorporation de l'acide aminé radioactif.

Cependant, la carence ou l'absence d'un élément nutritif indispensable provoque une diminution de l'appétit de l'animal ce qui entraîne une réduction de la consommation totale d'acides aminés indispensables, de protéine et d'énergie. La carence étudiée est donc la résultante de ces trois causes. Comme nous venons de le voir ci-dessus, les carences en protéines et en acides aminés inđispensables ont été étudiées par divers auteurs.

L'influence d'une diminution de la teneur en énergie de la ration sur la croissance du foie et du muscle du rat a fait l'objet de peu d'études : Durand et FaUconNEAU (I967) ont montré, en comparant des rats témoins et des rats restreints de même poids, que la concentration en protéines est augmentée tandis que celle d'ARN est diminuée.

Le but du présent travail est d'étudier à l'aide d'un acide aminé marqué, la synthèse protéique in vivo dans différents tissus du rat en croissance soumis à une réduction de l'apport énergétique de la ration. Dans une précédente publication (ARNAI, et FAUCONNEAU, I97I), les synthèses protéiques des différents tissus ont été déjà analysées chez le rat nourri ad libitum.

\section{MATÉRIEL ET MÉTHODES}

\section{1. - Matériel animal}

Des rats mâles de souche "Wistar " sont élevés dans des locaux éclairés pendant I 2 heures par jour, maintenus à une température de $22^{\circ} \mathrm{C}$ et à un degré hygrométrique contrôlé (6o p. 100$)$. Ils sont sevrés vers $70 \mathrm{~g}$, puis reçoivent jusqu'à I Io-I $20 \mathrm{~g}$ un aliment du commerce. Ils sont alors nourris ad libitum avec le régime témoin (ARNAL et FAUCONNEAU, I97I) jusqu'à I6o g environ. 


\section{2. - Régime, consommation alimentaire et croissance}

A partir de ce poids, les animaux reçoivent un régime contenant $24 \mathrm{p}$. Ioo de $\mathrm{MA}(\mathrm{N} \times 6,25)$ par rapport à la matière sèche (tabl. I) Après une période d'adaptation à la restriction énergétique, les quantités ingérées en fonction du poids vif sont celles décrites par DURAND et FAUCONNEAU, 1967 ). De ce fait, les rats " restreints " reçoivent chaque jour à I 4 heures en un seul repas (le repas étant très vite consommé : $20 \mathrm{mn}$ environ), les mêmes quantités de protéines, de vitamines et de minéraux que les animaux témoins mais seulement la moitié des aliments uniquement énergétiques (glucides et lipides). La croissance moyenne obtenue est de $2,2 \mathrm{~g} / \mathrm{j}$ our.

\section{TABLEAU I}

\section{Composition du régime (matière sèche $\mathrm{g} / \mathrm{kg}$ ) des animaux restreints}

\begin{tabular}{|c|c|}
\hline Amidon de blé $\ldots \ldots \ldots \ldots \ldots \ldots \ldots$ & 202 \\
\hline Farine de poisson de Norvège . . . . . . . . . . & 304 \\
\hline Mëthionine .. & 2,4 \\
\hline Saccharose & 220 \\
\hline Huile d'arachide $\ldots \ldots \ldots \ldots \ldots \ldots \ldots \ldots \ldots \ldots$ & 92 \\
\hline Agar-Agar $\ldots \ldots \ldots \ldots \ldots \ldots \ldots \ldots \ldots$ & 40 \\
\hline Mélange minéral $\ldots \ldots \ldots \ldots \ldots \ldots \ldots \ldots$ & 110 \\
\hline Mélange vitaminique .. & 17 \\
\hline Biotine $(40 \mathrm{mg} / 1) \ldots$ & $10 \mathrm{ml}$ \\
\hline Choline (200 mg/1) & $10 \mathrm{ml}$ \\
\hline
\end{tabular}

\section{3. -Administration du traceur - Abattage et prélèvement}

Au poids de $200 \mathrm{~g}$ obtenu à l'âge de $75-80 \mathrm{j}$, les rats reçoivent une injection intrapéritonéale de Io $\mu \mathrm{Ci}$ et $\mathrm{L}$ lysine ${ }^{14} \mathrm{C}$ (U) (activité spécifique $\mathrm{I} 28,4 \mathrm{mCi} / \mathrm{mM}$ ), I heure après le debut du repas.

Ces animaux sont ensuite abattus à des temps variables après l'injection, à raison de trois animaux par point expérimental. Deux autres lots de rats servent à la mesure de la radioactivité excrétée dans l'urine et le gaz carbonique. Un autre lot est sacrifié sans injection de radionucléide.

Chaque rat reçoit, un quart d'heure avant l'abattage, une injection intrapéritonéale de nembutal. Le sang est prélevé par ponction intra-cardiaque. La cavité abdominale est ouverte, le tube digestif (du cardia au rectum) est prélevé et lavé par ıoo $\mathrm{ml}$ d'acide trichloracétique io p. Ioo. Le reste de l'appareil digestif est enlevé de la cavité abdominale ainsi que les reins. Ces derniers, le foie et la rate, sont morcelés dans du sérum physiologique à $0^{\circ} \mathrm{C}$ a fin d'éliminer le maximum de sang. Foie, reins, tube digestif vide et glandes annexes constituent le compartiment " appareil digestif ".

Le rat est entièrement dépouillé, les muscles des membres postérieurs sont disséqués. Il reste alors ce que nous appelons " la carcasse".

Excepté le sang et le contenu digestif, chaque compartiment est congelé dans l'azote liquide et conservé en sachets plastiques à $-20^{\circ} \mathrm{C}$.

\section{4. - Méthodes analytiques et mesure des synthèses protéiques}

L'ensemble de ces méthodes a déjà été décrit (ARnal et FAUConneau, r97I).

Les méthodes analytiques utilisées permettent la mesure de la radioactivité de la lysine-14C libre ou protéique dans chaque compartiment. Les agents d'extraction des acides aninés libres sont l'éthanol $82^{\circ}$ pour le sang et l'acide trichloracétique ro $\mathrm{p}$. Ioo pour les autres tissus.

La méthode de mesure des synthèses protéiques est celle décrite par VANDERMEERs et VANDERMEERS-PIRET (1967). La vitesse de synthèse des protéines est d'ordre zéro alors que la vitesse de dégradation et d'exportation est d'ordre $\mathrm{I}$, ce qui pernet d'écrire qu'à tout instant $t$ :

$$
\frac{\mathrm{dN}}{\mathrm{d} t}=\mathrm{K}_{s} a(t)-\mathrm{K}_{\mathbf{E}+\mathbf{D}} \mathrm{N}
$$

$\mathrm{N}=$ radioactivité des protéines d'I $\mathrm{g}$ de tissu au temps $t$ exprimée en $\mathrm{DPM} / \mathrm{g}$. 
$a(t)=$ radioactivité spécifique du précurseur (lysine libre) au temps $t$ exprimée en DPM/mg.

$\mathrm{K}_{s}=$ constante de vitesse d'incorporation du précurseur ( $\mathrm{mg}$ de lysine incorporée dans les protéines d'I g de tissu par unité de temps)

$\mathrm{K}_{\mathbf{E}+\mathbf{D}} \mathrm{N}=$ constante de vitesse d'exportation et de dégradation.

Si le temps $t$ auquel on considère l'incorporation est court, le terme $K_{\mathbf{E}+\mathbf{D}} \mathrm{N}$ de l'égalité (r) est faible et peut être négligé d'où la nouvelle égalité :

$$
\frac{\mathrm{dN}}{\mathrm{d} t}=\mathrm{K}_{s} \mathrm{a}(t)
$$

si l'on intègre de o à $t$ :

$$
\mathrm{K}_{\varepsilon}=\frac{\mathrm{N}}{\int_{0}^{t} \mathrm{a}(t) \mathrm{d} t}
$$

Compte tenu des restrictions déjà énoncées (ARnal et Fauconneau, I97I), connaissant la teneur en lysine du tissu, il est aisé de connaître la quantité de protéines synthétisées. La teneur en protéine du tissu rapporté à la quantité synthétisée par unité de temps nous donne le temps de renouvellement.

La quantité de protéines synthétisées dans l'ensemble d'un compartiment (peau par exemple) rapporté à la quantité totale de protéines contenues dans ce compartiment conduit à la vitesse de remplacement.

\section{RÉSSULTATS}

\section{I. - Expression des résultats}

Trois modes d'expression sont utilisés :

- le pour cent de la dose injectée.

- le rapport entre les désintégrations par minute (DPM), par gramme de tissu frais et la radioactivité injectée (en DPM) par gramme de poids vif $\left(\frac{\mathrm{DPM} / \mathrm{gt}}{\mathrm{RAI} / \mathrm{gp}}\right)$. Ce rapport est multiplié par Ioo, il est aussi appelé formule de BLACK (ARNAI, et FAUCONNEAU, I97I).

- la radioactivité spécifique (RAS), qui est le rapport entre la formule de $B_{L A C K}$ et la teneur en lysine ( $\mathrm{mg} / \mathrm{g}$ de tissu) de la fraction considérée. Ce rapport est multiplié par I ooo.

Le bilan entre la radioactivité injectée et la somme de celle retrouvée dans les divers compartiments étudiés (carcasse, muscles des pattes postérieures, peau, appareil digestif, contenu digestif, sang, urine et gaz carbonique) est effectué pour chaque point expérimenta1. Il est exprimé en p. Ioo de la radioactivité injectée. La radioactivité contenue dans les os des pattes postérieures et les lipides n'a pas été mesurée. Chez les animaux témoins, ce bilan est compris entre 75 et 85 p. Ioo de la dose injectée. Une plus grande variabilité est obtenue chez les animaux restreints et nous avons effectué pour 4 points expérimentaux : $60 \mathrm{mn}, 480 \mathrm{mn}, 800 \mathrm{mn}$, I Ioo $\mathrm{mn}$, des transformations de données en utilisant les coefficients respectifs suivants $0,77, I, 33, I, 35, I, 4 \mathrm{I}$ pour comparer les résultats des divers points expérimentaux avec des bilans homogènes de radioactivité.

\section{2. - Teneur en lysine libre des différents compartiments}

Dans les quatre tissus ou organes étudiés et en particulier pour le sang et l'appareil digestif, la teneur en lysine libre est augmentée par la carence énergétique. 
SYNTHÈSE PROTÉIQUE ET CARENCE ÉNERGÉTIQUE

TABLEAU 2

Teneur en lysine libre tissulaire (mg/roo $\mathrm{g}$ poids frais)

\begin{tabular}{|c|c|c|c|c|c|}
\hline \multicolumn{2}{|c|}{ Temps (mn) } & 30 & 60 & 120 & 240 \\
\hline \multirow{2}{*}{ Muscle } & $\mathrm{T}(\mathbf{1})$ & 16,5 & 28,3 & 40,9 & 16,3 \\
\hline & $R\left({ }^{2}\right)$ & 22,8 & 31,0 & 32,0 & 27,5 \\
\hline \multirow{2}{*}{ Peau } & $\mathrm{T}$ & 11,7 & & 13,3 & 14,9 \\
\hline & $\mathbf{R}$ & 14,95 & 14,6 & 15,3 & 15,8 \\
\hline \multirow{2}{*}{ Appareil digestif } & $T$ & 6,9 & 8,3 & 7,3 & 7,9 \\
\hline & $\mathbf{R}$ & 12,3 & 11,7 & 9,6 & 9,2 \\
\hline \multirow{2}{*}{ Sang } & $\mathrm{T}$ & 3,7 & 5,9 & 5,45 & 4,2 \\
\hline & $\mathrm{R}$ & 7,3 & 5,7 & 10,5 & 6,0 \\
\hline
\end{tabular}

(1) Témoins.

(2) Restreints.

TABLEAU 3

Évolution de la L-lysine-14C libre tissulaive

\begin{tabular}{|c|c|c|c|c|c|c|c|c|c|}
\hline \multirow{2}{*}{\multicolumn{2}{|c|}{$\frac{\text { Expression des résultats }}{\text { Temps }(\mathrm{mn})}$}} & \multicolumn{4}{|c|}{$\frac{\mathrm{DPM} / g t}{\mathrm{RAI} / g p} 10^{2}$} & \multicolumn{4}{|c|}{ RAS $10^{3}$} \\
\hline & & 30 & 60 & 120 & 240 & 30 & 60 & 120 & 240 \\
\hline \multirow{2}{*}{ Sang } & $T\left({ }^{1}\right)$ & 13,2 & 12,2 & 9,7 & 1,4 & 3560 & 2080 & 1780 & 335 \\
\hline & $\mathrm{R}\left({ }^{2}\right)$ & 20,1 & 11,65 & 8,0 & 3,6 & 2750 & 2050 & 760 & 600 \\
\hline \multirow{2}{*}{ Muscle } & $\mathrm{T}$ & 41,4 & 56,1 & 36,8 & 8,2 & 2510 & 1970 & 900 & 500 \\
\hline & $\mathbf{R}$ & 49,0 & 45,5 & 35,1 & 18,1 & 2150 & 1460 & 1100 & 660 \\
\hline \multirow{2}{*}{ Peau } & $\mathrm{T}$ & 27,8 & & 7,6 & 1,9 & 2360 & & 570 & 130 \\
\hline & $\mathbf{R}$ & 28,75 & 14,8 & 11,3 & 6,55 & 1920 & 990 & 740 & 415 \\
\hline \multirow{2}{*}{ Appareil digestif } & $\mathrm{T}$ & 22,7 & 7,4 & 8,2 & 2,6 & $\mathbf{3} \mathbf{3 0 0}$ & 890 & 1130 & 330 \\
\hline & $\mathrm{R}$ & 24,3 & 12,75 & 5,5 & 4 & 1970 & 1080 & 570 & 410 \\
\hline
\end{tabular}

(1) Témoins.

(2) Restreints. 


\section{3. - Evolution de la radioactivité incorporée dans la L-lysine-14C libre des differents compartiments (tabl. 3)}

L'évolution de la radioactivité de la L-lysine-14C libre tissulaire n'est pas significativement différente pour les deux catégories d'animaux. Toutefois, au temps $240 \mathrm{mn}$, la valeur de la formule de Black est plus élevée dans les divers tissus ou organes chez les animaux restreints que chez les témoins : environ $2,5,2,5,3,5$ et $\mathrm{I}, 5$ fois plus, respectivement pour le sang, le muscle, la peau et l'appareil digestif.

La quantité de L-lysine- ${ }^{14} \mathrm{C}$ libre par gramme de tissu est toujours beaucoup plus élevée dans le muscle que dans le sang, 4 fois plus environ au temps $60 \mathrm{mn}$ après l'injection.

La radioactivité spécifique de la $\mathrm{L}-\mathrm{lysine}-{ }^{14} \mathrm{C}$ libre est plus faible $30 \mathrm{mn}$ après l'injection chez les rats restreints que chez les témoins de 20 p. IOO, I5 p. IOO, I5 p. IOO et $40 \mathrm{p}$. Ioo respectivement pour le sang, le muscle, la peau, et l'appareil digestif, ce qui est liéà la dilutionde la lysine injectée par une plus grande quantité de lysine libre non marquée. Les diverses remarques notées chez les témoins concernant l'évolution de la radioactivité spécifique (ARNAL et FAUCONNEAU, I97I) restent valables; en effet, les décroissances de la RAS de l'appareil digestif et de la peau sont plus rapides que celles du sang et du muscle. Comme les valeurs exprimées avec la formule de Black, les RAS sont plusélevées $240 \mathrm{mn}$ après l'injection chez les restreints que chez les témoins dans les différents compartiments. La lysine libre n'a pas été séparée dans les fractions acidosolubles des contenus digestifs.

\section{4. - Catabolisme de la L-lysine-14 $\mathrm{C}$}

\section{a) Catabolisme oxydatif}

La quantité de radioactivité dégagée sous forme de ${ }^{14} \mathrm{CO}_{2} 8$ heures après l'injection est égale à 25,7 p. Ioo de la dose injectée. Elle est environ 3 à 4 fois supérieure à celle obtenue chez les témoins pendant le même temps (7 p. Ioo de la dose injectée).

\section{b) Radioactivité de l'urine}

La radioactivité excrétée dans l'urine est faible et ne représente que 3,25 p. Ioo de la dose injectée un jour après l'injection. Cette valeur est assez voisine de celle trouvée chez les témoins (2,7 p. Ioo de la dose injectée). L'analyse radiochromatographique met en évidence de nombreux composés radioactifs que nous n'avons pas identifiés. Comme chez les témoins, l'urine ne contient que des traces de lysine non dosables.

\section{5. - L-lysine-14C incorporée dans les protéines}

\section{a) Sang (fig. I)}

L'incorporation de la lysine-14 $\mathrm{C}$ dans les protéines sanguines est très ralentie chez les restreints. $30 \mathrm{mn}$ après 1 'injection, la concentration en $1 y \operatorname{sine}{ }^{-14} \mathrm{C}$ des protéines sanguines n'est que la moitié de celle des rats témoins (fig. I). Dès $240 \mathrm{mn}$, la concentration en lysine-14 $\mathrm{C}$ semble avoir atteint un plateau relativement stable jusque vers 
les I $500 \mathrm{mn}$ après l'injection. Ce maximum d'incorporation est atteint beaucoup plus tôt que chez les témoins (480 mn après l'injection), la concentration en lysine-14 $\mathrm{C}$ correspondant à ce plateau n'atteint que les $35 \mathrm{p}$. Ioo de celle des témoins.

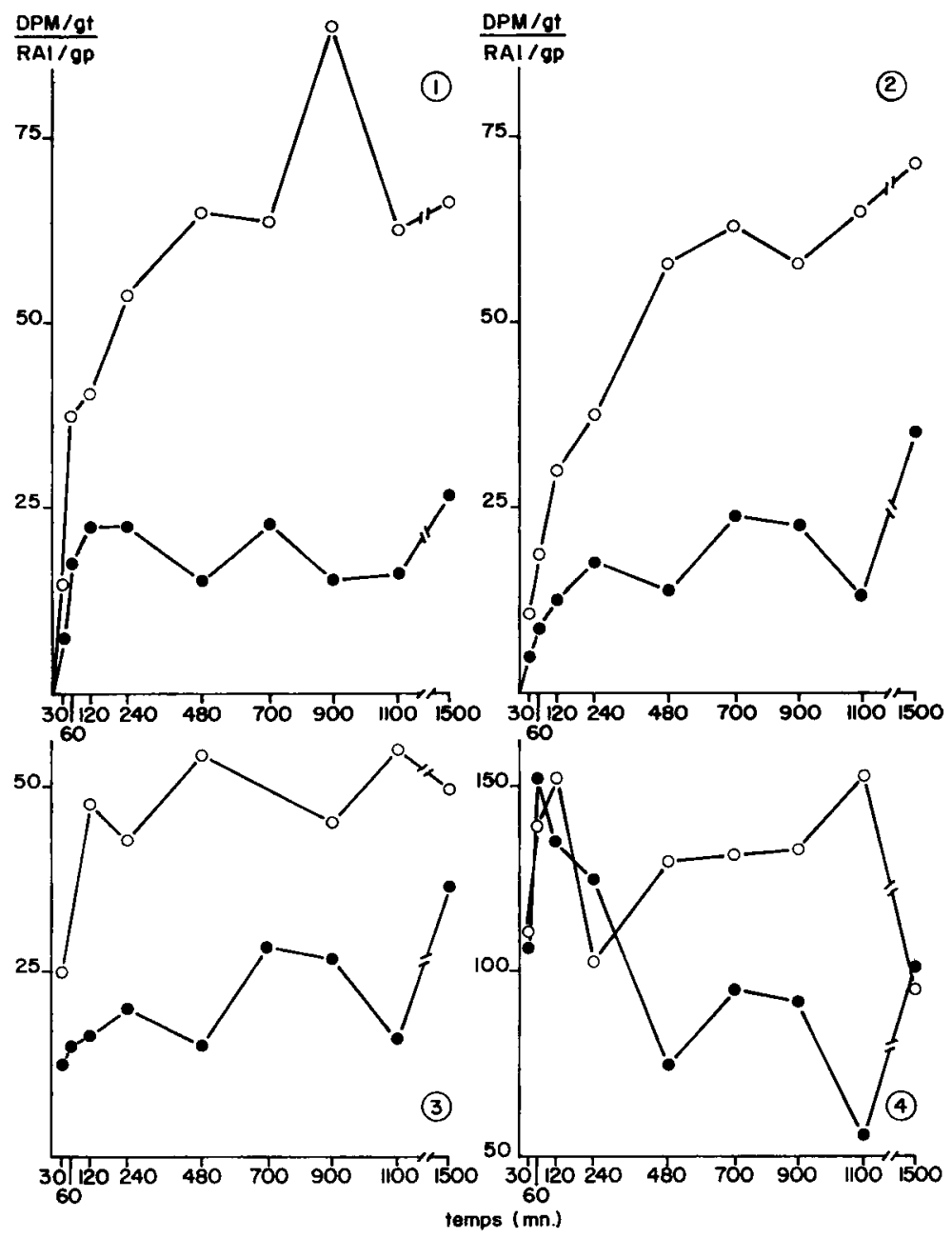

FrG. 1. - Évolution de la radioactivité éthanolo-insoluble du sang

FIG. 2. - Évolution de la radioactivité acido-insoluble du muscle

FIG. 3. - Évolution de la radioactivité acido-insoluble de la peau

Frg. 4. - Evolution de la radioactivité acido-insoluble de l'appareil digestif

○-— Témoins

- Restreints

b) Muscle (fig. 2)

Les mêmes constatations peuvent être formulées pour les protéines musculaires. On observe une diminution de la vitesse d'incorporation chez les animaux restreints et le maximum de concentration en lysine ${ }^{-14} \mathrm{C}$ est atteint plus tôt que chez les témoins. 
La quantité de radioactivité incorporée dans la masse musculaire totale est égale à Io p. Ioo de la dose injectée chez les restreints et $25 \mathrm{p}$. Ioo chez les témoins $700 \mathrm{mn}$ après 1 'injection.

\section{c) Peau (fig. 3)}

L'évolution de 1 'incorporation de la lysine- ${ }^{14} \mathrm{C}$ dans les protéines de la peau reste semblable à celle qui a été décrite ci-dessus pour le sang et le muscle.

d) Appareil digestif (fig. 4).

$30 \mathrm{mn}$ après 1 'injection, les concentrations delysine- ${ }^{-C} \mathrm{C}$ dans les protéines de 1'appareil digestif sont identiques chez les témoins et chez les restreints.

Jusqu'au temps $240 \mathrm{mn}$, les concentrations de lysine- ${ }^{14} \mathrm{C}$ dans les protéines ne sont pas significativement différentes. A partir de $480 \mathrm{mn}$, la quantité de $1 \mathrm{ysine}-{ }^{-14} \mathrm{C}$ incorporée dans les protéines augmente chez les témoins et continue à décroître chez les restreints. Au temps I $500 \mathrm{mn}$, les concentrations de lysine- ${ }^{14} \mathrm{C}$ ne sont plus significativement différentes. Cependant, comme les protéines de l'appareil digestif ont un " turnover " rapide, des variations importantes de radioactivité incorporée peuvent se produire en des temps très courts ; il est possible que le choix de nos points expérimentaux nous conduise à formuler ce résultat.

e) Contenu digestif (fig. 5)

L'évolution de la radioactivité de la fraction acido-insoluble du contenu digestif des animaux restreints est caractérisée par deux maxima atteints 480 et I $100 \mathrm{mn}$ après l'injection. Ils représentent respectivement 9,5 et $6 \mathrm{p}$. Ioo de la dose injectée. Chez les témoins, il existe un seul maximum, obtenu plus tôt, (au temps $240 \mathrm{mn}$ ) et sa valeur n'est égale qu'au tiers du maximum correspondant des restreints.
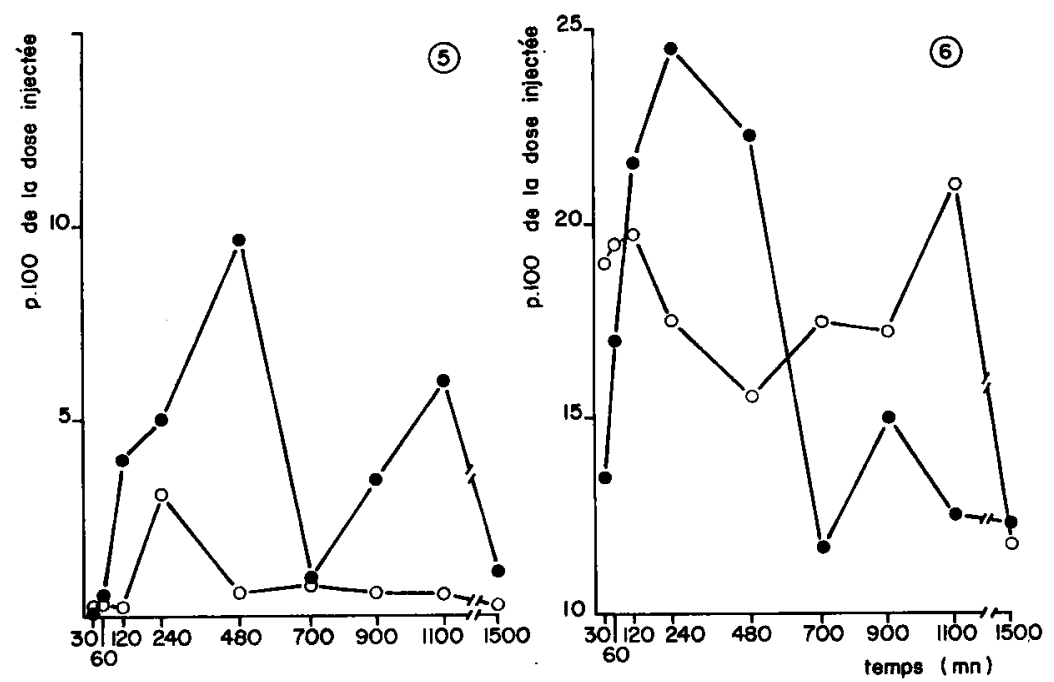

Fig. 5. - Évolution de la radioactivité acido-insoluble du contenu digestif

Fic. 6. - Evolution de la radioactivité totale de la somme-appareil digestif + contenu digestif

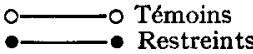


I a radioactivité totale captée par l'ensemble du contenu digestif et de l'appareil digestif (fig. 6) est plus élevée chez les restreints ayant reçu le repas I heure avant l'injection, que chez les témoins entre les temps r2o et $480 \mathrm{mn}$ ( $+35 \mathrm{p}$. Ioo au temps $240 \mathrm{mn}$ ) bien que chez les restreints l'appareil digestif soit plus petit $(9,5 \mathrm{p}$. Ioo du poids vif contre II p. Ioo pour les témoins).

\section{6. - Temps de renouvellement (tab1. 4)}

Chez les animaux restreints, les temps de renouvellement des protéines du muscle et de la peau sont plus élevés (multipliés par 2,I et 2,5 respectivement) alors que celui de l'appareil digestif est diminué de moitié.

TABLEAU 4

Temps de renouvellements (jours)

\begin{tabular}{|c|c|c|}
\hline & Témoins & Restreints \\
\hline Muscle & 7,5 & 16,1 \\
\hline Peau & 2,6 & 6,4 \\
\hline Appareil digestif & 1,06 & 0,56 \\
\hline
\end{tabular}

\section{7. - Protéines synthétisées (tabl. 5)}

Chez les restreints, les synthèses réelles dans les muscles, la peau et l'appareil digestif, représentent respectivement 60,50 et $200 \mathrm{p}$. Ioo de celles obtenues chez les témoins.

Pour l'ensemble des compartiments étudiés, la synthèse réelle totale est voisine dans les deux catégories d'animaux. Mais la quantité de protéine fixée étant plus faible chez les carencés en énergie, le rapport des protéines synthétisées aux protéines fixées est 2,5 fois plus élevé chez ces animaux.

\section{DISCUSSION}

\section{I. - Critique de la méthode}

Les limites de la méthode utilisée ont été analysées précédemment (ARNAL, et FaUCONNEAU, I97I).

Comme l'ont très bien décrit Vandermeers et VandermeErs-Pire'r ( 1967$)$, le calcul de la vitesse de biosynthèse fournit une valeur constante lorsqu'il est effectué en utilisant les valeurs de radioactivité protéique et libre obtenues à divers temps après l'injection, à condition que ce temps ne soit pas trop long. Il doit être inférieur à $20 \mathrm{mn}$ dans le cas du foie, d'après ces mêmes auteurs. La même constatation est effectuée pour les vitesses de biosynthèses de chaque compartiment, comme le montre le tableau 6. 
M. ARNAL, G. FAUCONNEAU, R. PECH

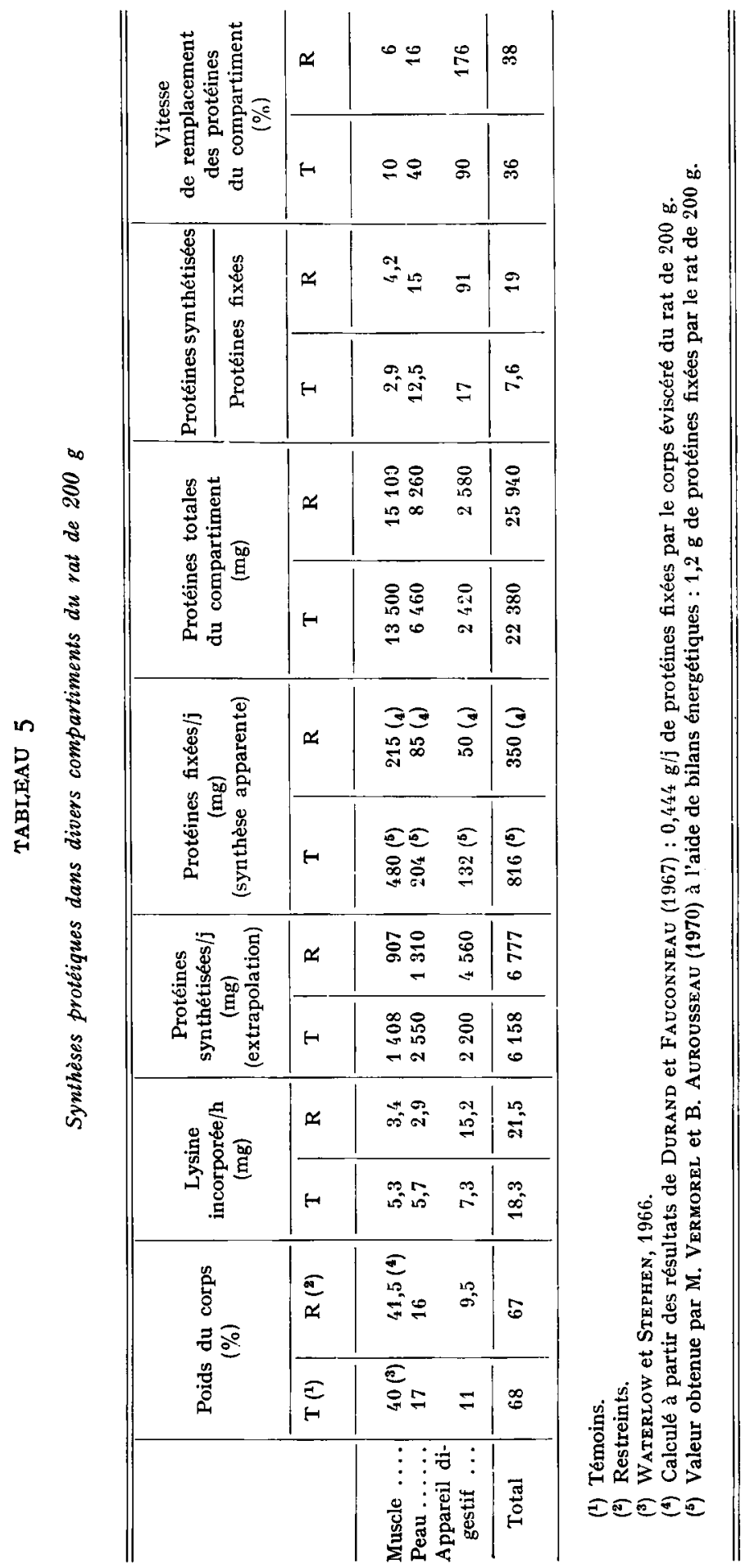


L,e temps de renouvellement obtenu à partir de la vitesse de synthèse des protéines (voir méthode) suit la même règle de validité. Le tableau 7 montre, que chez les animaux témoins, les estimations du temps de renouvellement des protéines musculaires

TABLEAU 6

Valeur du $K_{S}$ (mg de protéines/heure/g de tissu) en fonction du temps utilisé pour le calcul dans les différents compartiments

\begin{tabular}{|c|c|c|c|c|c|c|}
\hline \multicolumn{2}{|c|}{ Temps (mn) } & 30 & 60 & 120 & 240 & 480 \\
\hline \multirow{2}{*}{ Muscle } & $T\left({ }^{1}\right)$ & 0,810 & 0,86 & 0,95 & 0,6 & 1,0 \\
\hline & $R\left({ }^{2}\right)$ & 0,45 & 0,46 & 0,28 & 0,3 & \\
\hline \multirow{2}{*}{ Peau } & $T$ & 3,1 & & $2,,^{\prime}$ & & \\
\hline & $\mathbf{R}$ & 1,7 & 0,68 & 0,4 & & \\
\hline \multirow{2}{*}{ Appareil digestif } & $\mathrm{T}$ & 4,3 & 4,0 & 1,9 & & \\
\hline & $\mathbf{R}$ & 10,0 & 7,95 & & & \\
\hline
\end{tabular}

(1) Témoins.

(2) Restreints.

\section{TABLEAU 7}

Valeurs des temps de renouvellement (jours) en fonction du temps utilisé pour le calcul dans les différents compartiments

\begin{tabular}{|c|c|c|c|c|c|c|}
\hline \multicolumn{2}{|c|}{ Temps (mn) } & 30 & 60 & 120 & 240 & 480 \\
\hline \multirow{2}{*}{ Muscle } & $T\left({ }^{1}\right)$ & 8,3 & 7,8 & 7,2 & 11,3 & 6,5 \\
\hline & $R\left({ }^{2}\right)$ & 16,2 & 15,9 & 26,5 & 23 & \\
\hline \multirow{2}{*}{ Peau } & $\mathbf{T}$ & 2,6 & & 3,25 & & \\
\hline & $\mathbf{R}$ & 6,4 & 15,8 & 27,2 & & \\
\hline \multirow{2}{*}{ Appareil digestif } & $T$ & 1,06 & 1,1 & 2,4 & & \\
\hline & $\mathbf{R}$ & 0,56 & 0,7 & & & \\
\hline
\end{tabular}

(1) Témoins.

(2) Restreints.

ne sont pas significativement différentes jusqu'au temps $480 \mathrm{mn}$ après l'injection ; chez les restreints, une valeur constante ne peut être obtenue que pendant $60 \mathrm{mn}$. De même pour la peau, une valeur de l'ordre de 3 jours peut être calculée jusqu'au temps 
I20 $\mathrm{mn}$ après l'injection chez les témoins alors que chez les restreints, dès $60 \mathrm{mn}$, une valeur double de celle du temps $30 \mathrm{mn}$ est déjà calculée. La même remarque peut être effectuée pour l'appareil digestif. Il est donc possible que le premier point expérimental choisi $30 \mathrm{mn}$ après l'injection soit déjà un temps trop long et que chez les animaux carencés en énergie les valeurs de 6,4 et 0,56 jours pour la peau et 1'appareil digestif soient élevées.

L,e comportement alimentaire très différent des animaux témoins et restreints doit être considéré dans l'interprétation des résultats. L_es animaux restreints ingèrent en une vingtaine de minutes une quantité d'aliment égale à la moitié environ de celle que consomment en une jounée les animaux témoins. Or, chez les animaux carencés en énergie, l'injection de lysine- ${ }^{14} \mathrm{C}$ est effectuée pendant une période d'apport massif de nutriments ce qui peut entraîner un fonctionnement très accéléré du métabolisme protéique dans les organes régulateurs des viscères abdominaux. De plus, d'après les travaux de Rogers et HARPER (I966), la vidange stomacale est ralentie chez le rat après ingestion d'un repas important, d'où un apport de nutriment étalé dans le temps. Ces deux conséquences du comportement alimentaire peuvent expliquer le fait que, d'une part, au niveau de l'appareil digestif la décroissance de la radioactivité de la $1 y$ sine ${ }^{-14} \mathrm{C}$ des protéines entre les temps 60 et $240 \mathrm{mn}$ après 1 'injection est moins rapide chez les animaux restreints que chez les témoins, et d'autre part, que dans le contenu digestif le maximum de radioactivité rencontré au temps $240 \mathrm{mn}$ chez les témoins se situe plus tard chez les animaux carencés en énergie, au temps $480 \mathrm{mn}$.

\section{2. - Teneur en lysine libre et pénétration de la lysin3-14C libre dans les divers compartiments}

Les teneurs élevées de lysine libre du muscle et du sang chez les animaux carencés en énergie sont à relier avec la propriété particulière de cet acide aminé que constitue sa possibilité d'accumulation sous forme libre dans ces deux tissus (le tissu musculaire en particulier) lorsque la teneur en lysine de la ration augmente (PAWLAK et ProN, I968). Cependant, compte tenu d'une part que, comparée aux résultats de ces mêmes auteurs, $27,9 \mathrm{mg}$ de lysine par Ioo $\mathrm{g}$ de muscle obtenus avec une consommation de $225 \mathrm{mg}$ de lysine par jour, et d'autre part que les animaux témoins et restreints ont ingéré approximativement la même quantité de lysine soit $2 \mathrm{I}_{4}$ et $204 \mathrm{mg}$ par jour respectivement au poids de $200 \mathrm{~g}$, la teneur en lysine du muscle des restreints, $30,2 \mathrm{mg}$ par roo g de muscle, représente une faible augmentation.

Les besoins pour les synthèses protéiques, dans les tissus ou organes étudiés, qui représentent $68 \mathrm{p}$. Ioo du poids du corps, sont légèrement plus élevés pour les restreints (tabl. 5). Trois à quatre fois plus de lysine environ est utilisée à des fins énergétiques chez les restreints comme en témoigne la quantité de gaz carbonique marqué dégagée en 8 heures. Pour relier ces faits à une teneur plus élevée de lysine dans tous les tissus, on peut supposer qu'un des mécanismes d'adaptation à la réduction énergétique est identique à celui décrit par STEPHEN et WATERLOW (rg66) dans le foie de rat recevant un régime à faible taux azoté. Il y aurait une augmentation de la réutilisation des acides aminés dans 1'appareil digestif ; en effet, c'est la valeur élevée des synthèses protéiques dans ce compartiment qui compense la diminution des synthèses dans le muscle et dans la peau (voir discussion ci-dessous).

Le manque d'énergie ne semble pas affecter la pénétration de la lysine- ${ }^{14} \mathrm{C}$ dans 
le principal réservoir d'acides aminés libres qu'est le muscle. $60 \mathrm{mn}$ après l'injection, la réserve de lysine- ${ }^{14} \mathrm{C}$ libre musculaire représente dans les deux catégories d'animaux 22 p. roo de la dose injectée. En revanche, elle semble y rester plus longtemps puisque $240 \mathrm{mn}$ après l'injection, la RAS et la formule de Black ont des valeurs plus élevées chez les restreints que chez les témoins. La concentration en lysine dépendant de la vitesse d'entrée et de sortie, d'une part, et de l'anabolisme et du catabolisme, d'autre part, il est difficile de savoir lequel de ces quatre facteurs est responsable de ce séjour plus élevé de la lysine dans le muscle.

L'effet de la carence énergétique est diffárent de celui de la carence azotée, GAETANI et al. ( 1963 ) montrent en effet, que chez des rats recevant un régime protéiprive, la vitesse de pénétration de la lysine- ${ }^{14} \mathrm{C}$ est diminuée dans le foie et le muscle gastrocnémien, le foie étant plus affecté que le muscle. Le critère utilisé est la valeur du rapport entre la radioactivité spécifique de la lysine- ${ }^{14} \mathrm{C}$ libre dans le tissu et celle du même acide aminé dans le plasma. Ces auteurs observent un rapport plus petit chez les animaux en carence azotée que chez les témoins.

\section{3. - Incorporation de la $L$-lysine-14C dans les protéines des divers compartiments}

La distribution de la radioactivité dans les protéines des différents compartiments est très affectée par la réduction énergétique (fig. I, 2, 3, 4) : alors que les quantités de $1 y$ sine- ${ }^{14} \mathrm{C}$ incorporées dans le muscle et la peau sont fortement diminuées (50 p. Ioo environ), celles de l'appareil digestif ne sont pas différentes pendant $240 \mathrm{mn}$ pour les deux catégories de rats.

Cette prédominance du marquage des viscères, et en particulier du foie, lors de l'emploi d'acides aminés radioactifs, a été montrée chez le rat nourri avec un régime à faible taux protéique (6 p. Ioo de caséine) par WATERLOW et STEPHEN (I966). GaETANI et al. (I963) avec un régime protéiprive montrent que l'activité spécifique des protéines du foie des rats carencés est 3 fois plus élevée que celle des témoins une heure après l'injection de Io $\mu \mathrm{Ci}$ de $\mathrm{DL}$ lysine- ${ }^{14} \mathrm{C}$ par Ioog de poids vif tandis que celle des protéines musculaires est égale à 75 p. Ioo de la valeur obtenue chez les témoins. De même SidRANSKY et WAGLE (I 669 ), chez des rats nourris par ingestion forcée d'une ration sans thréonine, trouvent qu'une heure après injection intrapéritonéale de leucine ${ }^{14} \mathrm{C}$, la radioactivité spécifique des protéines hépatiques est 37 p. roo plus élevée que chez les témoins recevant de la même manière un régime complet mais que, pendant la même durée, les protéines musculaires ont incorporé moins de leucine- ${ }^{14} \mathrm{C}$ (- 33 p. IOo).

Les effets d'une carence énergétique ou azotée (qualitative ou quantitative) sur la distribution de la radioactivité dans l'organisme après injection d'un acide aminé marqué sont semblables.

\section{a) Muscle}

\section{4. - Synthèse protéique}

L'anabolisme réel des muscles est diminué de $40 \mathrm{p}$. roo par la carence énergétique. Comme le muscle est plus riche en protéines (DuRAND et FAuconneau, I967), la synthèse des protéines est encore plus diminuée lorsqu'elle est exprimée par 
gramme de tissu (- 45 p. roo). Aussi, le temps de renouvellement des protéines musculaires double à la suite de la réduction de l'apport énergétique de la ration.

C'est aussi la constatation de WATERLow et STEPHEN (I968), dans le cas d'une infusion continue de $\mathrm{L}$-lysine- ${ }^{14} \mathrm{C}$ à des rats recevant un régime à faible taux protéique.

Le catabolisme des protéines, apprécié par la différence entre les protéines réellement synthétisées et les protéines fixées, accuse une baisse moins importante que la synthèse (- 25 p. IOO). Malgré cela, le rendement de fixation des protéines (rapport entre 1'anabolisme apparent et 1'anabolisme réel), qui était de $34 \mathrm{p}$. Ioo chez les témoins, n'est plus que de $24 \mathrm{p}$. Ioo chez les restreints. La carence énergétique a donc abaissé le rendement de fabrication des protéines musculaires de $30 \mathrm{p}$. Ioo environ.

- La confrontation de ces résultats avec les teneurs en acides nucléiques, (DURAND et FAUCONNEAU, I967), montre que la carence énergétique entraîne une diminution de la synthèse protéique de $40 \mathrm{p}$. Ioo associée avec une diminution de la quantité d'ARN par unité d'ADN de 26 p. I0o, alors que la quantité totale d'ADN est 20 p. IOO plus faible et que I9 $\mathrm{p}$. Ioo de protéines en plus sont obtenus par unité d'ADN. Dans l'expérience de ces auteurs, il est à noter que les rats étaient soumis à une réduction énergétique depuis le poids de $70 \mathrm{~g}$ jusqu'à $200 \mathrm{~g}$.

La carence énergétique agirait donc au niveau du muscle beaucoup plus par un ralentissement de la synthèse que par une augmentation du catabolisme.

\section{b) Peau}

La synthèse des protéines de la peau est encore plus affectée que celle du muscle, elle est égale chez les animaux restreints à la moitié de celle obtenue chez les témoins. La carence énergétique a des répercussions identiques sur l'anabolisme et le catabolisme dans ce tissu. Le temps de renouvellement des protéines est triplé car, comme dans le muscle, la teneur en protéines est de $20 \mathrm{p}$. Ioo environ supérieure à celle des témoins.

Ces résultats sont à rapprocher du fait que les protéines de la peau, tissu représentant 25 à 30 p. roo de l'azote total chez le rat, sont facilement mobilisables en cas de carence énergétique (DURAND et PENOT, I969). La participation de la peau à la perte totale d'azote, due à l'apport d'un régime carencé en protéines (6 p. roo de caséine) pendant 5 à 8 semaines, à des rats, est de 34,5 p. roo, alors que celle-ci est de 25,9 p. IOo pour les muscles et I4,5 p. IOO pour les viscères (WATERLOW et STEPHEN, I966).

\section{c) Appareil digestif}

A poids d'animal égal, l'appareil digestif tel que nous l'avons défini (voir méthode) est plus léger chez les restreints (- I5 p. I00). Ceci est en bon accord avec les résultats de DURAND et FAUCONNEAU ( 1967 ) qui trouvent que le foie des rats restreints en énergie depuis le poids de $70 \mathrm{~g}$ jusqu’à $200 \mathrm{~g}$, a un poids inférieur de $29 \mathrm{p}$. roo.

A l'opposé des phénomènes observés dans le muscle et la peau, les synthèses protéiques sont doublées dans l'appareil digestif et l'anabolisme protéique rapporté au gramme de tissu est fortement augmenté (+67 p. I00). Il en est de même pour le catabolisme. Le rapport des protéines fixées sur les protéines synthétisées atteint 
des valeurs très faibles : $0,1 \mathrm{p}$. Ioo chez les restreints contre $6 \mathrm{p}$. Ioo chez les témoins. En conséquence, la carence énergétique diminue de moitié le temps de renouvellement des protéines de ce compartiment.

Des augmentations de cet ordre de grandeur ont été trouvées par VANDERMEERS et VANDERMEERS-PIRET (I967) à la suite d'une carence en trois acides aminés indispensables : la lysine, la thréonine et le tryptophane chez le rat (régime gluten). Ces auteurs signalent des augmentations de synthèses protéiques respectivement de 4 I et IOI p. Ioo dans le foie et le pancréas de ces animaux.

La carence azotée et la carence énergétique ont, semble-t-il, des effets analogues sur la synthèse des protéines au niveau des viscères abdominaux.

Pour étudier la réutilisation des acides aminés dans différents tissus, STEPHEN et WATERI,OW (rg66) ont utilisé l'arginine marquée sur le carbone-I et le carbone-6, et GAETANI et al. (I964) ont déterminé les concentrations d'enzymes activateurs. des acides aminés dans le foie de rat soumis à une carence azotée. Ces auteurs ont trouvé une augmentation de la réutilisation des acides aminés aux sites de synthèses. Ainsi un acide aminé libre tel que la $1 y \operatorname{sine}{ }^{-14} \mathrm{C}$ provenant du catabolisme protéique a une probabilité plus élevée d'être réincorporé dans les protéines, chez les animaux restreints en énergie d'où une augmentation de la vitesse de renouvellement.

\section{d) Protéines synthétisées dans l'ensemble des compartiments étudiés}

La quantité de protéines synthétisées dans 1'ensemble muscle, peau et appareil digestif (qui représente le même pourcentage du poids vif, environ $67 \mathrm{p}$. Ioo dans les deux catégories d'animaux) est légèrement plus élevée chez les restreints que chez les témoins $(6,7 \mathrm{~g}$ par jour contre $6, \mathrm{I} \mathrm{g})$. Il n'y a pas de réduction de la synthèse protéique au cours de la carence énergétique dans l'ensemble des tissus considérés. Picou et TAyLor-RoBerTs (I969) par infusion intragastrique de glycine- ${ }^{15} \mathrm{~N}$ chez 1'enfant sous-alimenté trouvent des résultats analogues : $6,5 \mathrm{~g}$ de protéines $/ \mathrm{kg} / \mathrm{jour}$ chez les témoins contre $6,2 \mathrm{~g}$ protéines $/ \mathrm{kg} / \mathrm{jour}$ chez les enfants souffrant de malnutrition.

Le rendement des protéines fixées par rapport aux protéines synthétisées est, par contre, très diminué par la carence énergétique, il passe de $\mathrm{I} 3 \mathrm{p}$. Ioo à $5 \mathrm{p}$. Ioo, ceci étant lié au rendement très bas dans l'appareil digestif ( $\mathrm{I}$ p. IOo).

\section{e) Contenu digestif}

La quantité de radioactivité apparue dans les fractions acido-soluble et insoluble du contenu digestif est très importante. Les sommes des radioactivités de ces deux fractions sont approximativement égales dans le contenu digestif et l'appareil digestif : II,5 et Io,7 p. Ioo de la dose injectée respectivement au temps $480 \mathrm{mn}$. Pour ce même temps, la quantité de radioactivité du compartiment musculaire n'est que 7,7 p. Ioo de la dose injectée. En utilisant le même mode d'expression des résultats, la figure 6 montre que l'ensemble de l'appareil digestif et du contenu digestif a capté une quantité de lysine ${ }^{-14} \mathrm{C}$ plus élevée chez les restreints que chez les témoins entre les temps 60 et $700 \mathrm{mn}$ ( $+35 \mathrm{p}$. I00 au temps $240 \mathrm{mn}$ ).

Ces résultats sont en faveur d'une participation plus importante de l'azote endogène dans la régulation du métabolisme protéique lors d'une carence en énergie au moins dans la phase qui suit le repas. 


\title{
CONCLUSION
}

I1 existe un certain nombre d'analogies dans les mécanismes d'adaptation de l'animal à la carence azotée et à la carence énergétique, peu étudiée, qui fait l'objet de ce travail.

La carence en énergie, en présence d'un excès de protéines dans la ration, entraîne une utilisation énergétique de l'azote du régime; ainsi une partimportante de la lysine, acide aminé indispensable, pourtant peu catabolisable, a été utilisée à cette fin.

Les quantités totales de protéines synthétisées par l'animal ne sont pas affectées par le manque d'énergie. Mais, la part due à chaque compartiment met en évidence le rôle régulateur très important de l'appareil digestif qui, par une augmentation des synthèses, contrebalance les diminutions observées dans la peau et le muscle. La carence énergétique entraîne une élévation du temps de renouvellement des protéines du muscle et de la peau tandis que celui des protéines de l'appareil digestif est diminué.

La plus grande quantité de radioactivité apparue dans les contenus digestifs indique une participation plus élevée de l'azote endogène digestif à la régulation du métabolisme protéique en cas de carence énergétique. Ce résultat apparaît clairement lorsque $l^{\prime}$ injection de $1 y \operatorname{sine}-{ }^{14} \mathrm{C}$ est effectuée après le repas.

\section{Reçu pour publication en juillet 1971.}

\section{REMERCIEMENTS}

Nous remercions vivement J. Prugnaud pour les analyses radiochromatographiques de Lysine-14 C.

\author{
SUMMARY \\ PROTEIN SYNTHESIS IN VIVO IN DIFFERENT TISSUES OF \\ GROWING RATS SUBJECTED TO REDUCTION \\ OF ENERGX SUPPLIED BY THE RATION
}

Two groups of rats weighed about $160 \mathrm{~g}$ at the start of the experiment. Control groups were fed to appetite on a balanced diet with I4 p. cent crude protein $(N \times 6,25)$.

Restricted groups received a low energy diet with 24 p. cent crude protein $(N \times 6,25)$. This group was rationed so that daily intakes of protein, vitamines and minerals were the same of control group but energy was only half as much.

Daily gain was $6.0 \mathrm{~g}$ in controls and $2.2 \mathrm{~g}$ for the restricted rats. At $200 \mathrm{~g}$ body weight $10 \mu \mathrm{Ci}$ L-lysine-14 $\mathrm{C}$ was injected intraperitoneally and the rats were killed at different intervals after injection.

The rats were dissected into 6 compartments, blood, muscles of the hind limbs, digestive tract (empty digestive tract from cardia to rectum and abdominal viscera including kidneys), contents of digestive-tract, skin and carcass. Radioactivity of the lysine-14 $\mathrm{C}$ was measured in the free amino acid and protein fractions of the different compartments. Protein synthesis was studied 
during a short time after injection in order to minimise the re-utilisation of the precursor on the one hand and taking account of the radioactivity of this precursor on the other.

- Free lysine content (table 2). Free lysine contents increased in the restricted rats.

- Specific radioactivity of free L-lysine-14C (table 3). It changed roughly in the same way in the 2 groups. During the first 2 hours muscle was a reserve of free lysine which later disappeared.

- Amount of radioactivity lost as ${ }^{14} \mathrm{CO}_{\mathrm{g}}$. It was 3 to 4 times as much $8 \mathrm{~h}$ after injection in the restricted rats, indicating that protein had been used as a source of energy.

- Incorporation of L-lysine-14C into proteins (fig. $1,2,3$ and 4 ) In restricted rats it was lower in muscle, skin and blood. It did not differ in the digestive tract during 240 minutes.

- Proteins synthesis (table 5). In muscle, skin and digestive tract of the restricted rats, values for protein synthesis were 60,50 and 200 per cent, respectively, of values in the controls. For these compartments combined the amounts of protein synthesized did not differ significantly between groups.

- Turnover time (table 4). Turnover time for protein of muscle was 7.5 days in the control rats and r6.I days in the restricted. For skin the respective times were 2.6 and 6.4 days and for the digestive tract 1.06 and 0.56 days.

- Contents of digestive tract (fig. 5 and 6). Radioactivity in acid-soluble and insoluble fractions was much more important in the restricted rats. Two maxima appeared, 480 and I roo minutes after injection.

The results are discussed. They show the importance of the abdominal viscera in the phenomena regulating protein metabolism during energy deficit.

\section{RÉFÉRENCES BIBLIOGRAPHIQUES}

Arnal M., Fauconneau G., Pech R., r97r. Étude de la synthèse protéique " in vivo ": mesure de l'activité anabolisante de divers tissus du rat en croissance à l'aide de la L lysine ${ }^{14} \mathrm{C}$ (U). Ann. Biol. anim. Bioch. Biophys., 11, 245-264.

Durand G., Fauconneau G., Penot E., Ig66. Croissance des tissus du rat et qualité des protéines alimentaires ; influence sur le nombre et la taille des cellules. Ann. Biol. anim. Bioch. Biophys., 6, 389409.

Durand G., Fauconneau G., Penot E., I967. Croissance des tissus du rat et réduction de l'apport énergétique de la ration; influence sur la teneur en acides nucléiques. Ann. Biol. anim. Bioch. Biophys., 7. I 45-I55.

Durand G., Penot E., I969. Évolution du nombre et de la taille des cellules dans les tissus de la ratte adulte amaigrie à la suite d'une carence énergétique. Croissance compensatrice. Ann. Biol. anim. Bioch. Biophys., 8, 575-587.

Gaetani S., Mariani A., Spadoni, M. A., Tomassi G., I963. Distribuzzione della ${ }^{14 C}$ lisina in tessuti di ratti in deplezione proteica. Quad. Nutr., 28, I-I2.

Gaetani S., Paolucci AM. M., Spadoni M. A., Tomassi G., 1964. Activity of aminoacid activating enzymes in tisues from protein depleted rats. J. Nutr., 84, I73-178.

MunRo H. N., 1964. General aspects of the regulation of proteins metabolism by diet and by hormones In Mammalian protein metabolism Ed. Munro H. N. Vol. I p. 381 . Academic press. New York.

Pawlak M., Pion R., I968. Influence d'une diminution du taux protéique de la ration sur les teneurs en acides aminés libres des tissus sanguin et musculaire du rat en croissance. Ann. Biol. anim. Bioch Biophys., 8, 457-459.

Picou D., Taylor-Roberts T., I969. The measurement of total protein synthesis and catabolism and nitrogen turnover in infants in different nutritional states and receiving different amount of dietay protein. Clin. Sci., 38, 283-296.

Rogers Q. R., Harper A. E., I966. Protein digestion : Nutritional and Metabolic considerations. Wld. Rev. Nutr. Diet., 6, 250-29I.

Sснгмке R. T., I964. The importace of both synthesis and degradation in the control of arginase levels in rat liver. J, Biol. chem., 239, 3808-38 7 .

SIDRANSKY H., WAGLE D.S., VERNEY E., I969. Hepatic protein synthesis in rats force-fed a threonine: devoid diet and treated with cortisone acetate or threonine. Lab. Invest., 20, 364*37o.

Sidransky H., Verney E., r970. Skeletal muscle protein metabolism changes in rats force fed a diet inducing an experimental kwashiorkor-like model. Am. J. Clin. Nutr., 23, I I54-I I59.

Stephen J. M. L., WAterlow J. C., I966. Use of carbon 44 labelled arginine to measure the catabolic: rate of serum and liver proteins and the exent of aminoacid recycling. Nature, 211, 978-980.

Vandermeers-Piret M. C., Christophe J., 1964. Retentissement d'une carence en lysine sur-le foire de rat. Arch. Internation. Physiol. Biochem., 72, 703-705. 
Vandermeers A., Vandermeers-Piret M. C., Christophe J., i967. Synthèses protéiques in vivo dans le pancréas et le foie du rat normal, carencé en lysine et en thréonine, ou récupérant de cette carence. Bull. Soc. chim. Biol., 49, 759-778.

Vermorel M., Aurousseau B., i97o. Utilisation énergétique des acides acétique et caprylique par le rat en croissance. In 5th Symposium on Energy metabolism of farm animals. Ed European Association for animal production. p. 185 Jarvis Druck, Verlag Zürich.

Waterlow J. C., Stephen J. M. L., Ig66. Adaptation of the rat to a low protein diet the effect of a reduced protein intake on the pattern of incorporation of $\mathrm{L}\left({ }^{14} \mathrm{C}\right)$ lysine. $B r . J . N u t r, 20,46 \mathrm{I}^{* 484}$.

Waterlow J. C., Stephen J.M. L., rg68. The effect of low protein diets on the turnover rates of serum, liver and muscle proteins in the rat, measured by continuous infusion of $\mathrm{L}\left({ }^{14} \mathrm{C}\right)$ lysine. Clin $\mathrm{Sci}$. 85 , 287-305.

Wannemacher R. W., I965. Plasma amino acid concentrations in relation to protein synthesis in protein nutrition and free amino acid patterns. In Protein nutrition and free amino acid patterns. Ed Leathem J. H. Rutgers university press; New Brunswick. p. 206. 